Pacific Journal of Mathematic 


\section{THE GEOMETRIC INTERPRETATION OF A SPECIAL CONNECTION}

\section{M. SINGER}

1. Introduction. An esthetic problem remaining in the foundations of Riemannian geometry is this. To each Riemannian metric on a manifold one associates a connection on its bundle of bases. This connection is uniquely characterized by its having zero torsion and its parallel translation preserving the metric. By very definition at each tangent space of the bundle the connection assigns a complement to the vertical. Yet the various existence proofs of the Riemannian connection are computational and do not exhibit this complementary space (the horizontal space) directly. The problem is how can one do so directly from the metric, given (say) by the reduction of the bundle to the bundle of frames.

Under rather special circumstances, we exhibit a geometric and coordinate free construction of a connection in $\$ 2$. This includes the class of complex analytic bundles and gives a geometric construction of a connection of type $(1,0)$ with curvature of type $(1,1)$, useful in algebraic geometry (§4). See [2] and [5]. For the case of complex vector bundles Nakano computes this connection in terms of coordinates [6]. For Kähler manifolds it turns out (\$2) that this connection is the Riemannian connection restricted to the unitary subbundle. Hence we obtain a solution to the problem posed in the preceding paragraph for Kähler manifolds.

We assume the reader is familiar with the bundle approach to connections, say as in [1]. We use the notation of that paper.

2. The connection. Let $\mathfrak{B}=(B, M, G, G, \pi, \Phi)$ be a principal bundle. Throughout this discussion we shall suppose that $G$ is the complexification of a compact group $^{1}$ (such is the case for example if $G$ is complex semisimple) and that $B$ is an almost complex manifold. If $b \in B$, let $J_{b}$ denote the linear transformation on $B_{b}$ (the tangent space of $B$ at $b$ ) giving the almost complex structure on $B$, so that $J_{b}^{2}=-I$. We assume the following two conditions on this almost complex structure:

(i) the structure is invariant under right translation by $G$, that is $R_{g} J_{b}=J_{b q} R_{g}$

Received July, 29, 1958. This research was supported in part by the United States Air Force under Contract No. AF 18 (603)-91 monitored by the Air Force Office of Scientific Research of the Air Research and Development Command.

1 The results of this paper are valid whenever the Lie algebra $g$ of $G$ is the complexification of the Lie algebra ${ }^{\ddagger}$ of $K$. The compactness of $K$ is assumed only to guarantee that the reduction to $K$ is possible. 
(ii) $J_{b}$ leaves the vertical subspace $V_{b}=\pi^{-1}(0)$ of $B_{b}$ invariant and therefore $J_{b}$ restricted to $V_{b}$ induces an almost complex structure on the fibre containing $b$. This almost complex structure should agree with the two sided invariant complex structure of $G$, when the fibre is identified with $G$.

One knows [7] that the structural group of $\mathfrak{B}$ can be reduced to the maximal compact subgroup $K$ of $G$. This fact implies the existence of a submanifold $B_{k}$ of $B$ passing through a fixed point $b \in B$, where $B_{k}$ is the underlying space of a subbundle $\mathfrak{B}_{k}=\left(B_{k}, M, K, K, \pi, \Phi^{\prime}\right)$ of $\mathfrak{B}$. The main observation is this

THEOREM. For every reduction of $\mathfrak{B}$ to a subbundle $\mathfrak{B}_{k}$, there exists a unique connection $H$ on $\mathfrak{B}$ satisfying the two conditions:

( $\alpha)$ If $c \in B, J_{c}\left(H_{c}\right)=H_{c}$, that is, the connection $H$ is invariant under the almost complex structure.

( $\beta)$ If $c \in B_{k}, H_{c} \subset\left(B_{k}\right)_{c}$, the subspace of $B_{c}$ tangent to $B_{k}$. This condition is equivalent to the statement that $H$ restricted to $B_{k}$ is a connection on the bundle $\mathfrak{B}_{k}$.

Proof. Since $G$ is the complexification of $K$, its Lie algebra $\mathfrak{g}$ is the complexification of the subalgebra $\mathfrak{f}$, the Lie algebra of $K$. Let $c$ be a point of $B_{k}$, and identify the fibre containing $c$ with $G$, so that $c$ becomes the identity of $G$. Then $V_{c}$ is identified with $g$ and under this

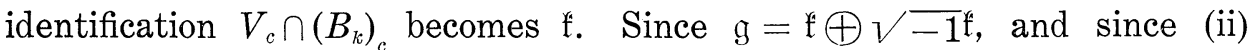
holds, we have $V_{c}=V \cap\left(B_{k}\right)_{c} \oplus J_{c}\left(V_{c} \cap\left(B_{k}\right)_{c}\right)$. To simplify the notation, let $W=\left(B_{k}\right)_{c}$ and $W^{\prime}=J_{c}\left(\left(B_{k}\right)_{c}\right)$, so that $V_{c}^{c}=V_{c} \cap W \oplus V_{c} \cap W^{\prime}$ because $J_{c}\left(V_{c}\right)=V_{c}$.

We define the horizontal space of our connection, $H_{c}$, to be $W \cap W^{\prime}$. We must show that $H_{c}$ is a linear complement to $V_{c}$ in $B_{c}$. $V_{c} \cap H_{c}=$ $V_{c} \cap W \cap W^{\prime}=\left(V_{c} \cap W\right) \cap\left(V_{c} \cap W^{\prime}\right)=0$ because $V_{c}=V_{c} \cap W \oplus V_{c} \cap W^{\prime}$. This shows that $H_{c}$ is a complement to $V_{c}$. To show that it is a full complement, we need only check that the dimension of $H_{c}$ equals the dimension of $M$. Let $\operatorname{dim}(M)=m$. Let $\operatorname{dim}(K)=n$, so that the real $\operatorname{dim}(G)=2 n, \operatorname{dim}(B)=2 n+m$, and $\operatorname{dim} W=\operatorname{dim} W^{\prime}=\operatorname{dim} B_{k}=n+m$. Since $\operatorname{dim}(W)+\operatorname{dim}\left(W^{\prime}\right)=\operatorname{dim}\left(W \cap W^{\prime}\right)+\operatorname{dim}\left(W \cup W^{\prime}\right)$, to prove $\operatorname{dim}\left(H_{c}\right)=m$ it suffices to show that $\operatorname{dim}\left(W \cup W^{\prime}\right)=2 n+m$. In fact, we show that $W \cup W^{\prime}=B_{c}$. If $t \in B_{c}$, then $\exists w \in W \ni \pi(w)=\pi(t)$ for $B_{k}$ is a bundle over $M$. Since $\pi(w-t)=0, w-t=v \in V_{c}$ and therefore $w=t+v \in W \cup V_{c}$; that is

$$
B_{c} \subset W \cup V_{c} \subset W \cup\left(V_{c} \cap W \oplus V_{c} \cap W^{\prime}\right) \subset W \cup W^{\prime} .
$$

As $c$ varies over $B_{k}$, the choice $H_{c}$ gives a connection on $\mathfrak{B}_{k}$. For $H_{c} \subset W$ and we have already shown that $H_{c}$ is a full complement to $V_{c}$ 
in $B_{c}$ and hence to $V_{c} \cap W$ (the vertical subspace of $\mathfrak{B}_{k}$ ) in $W$. Moreover $H_{c}$ is invariant under right translation by $k \in K$ by (i), that is

$$
\begin{aligned}
R_{k}\left(H_{c}\right) & =R_{k}\left(W \cap W^{\prime}\right) \\
& =R_{k}(W) \cap R_{k}\left(J_{c}(W)\right)=R_{k}(W) \cap J_{c k}\left(R_{k}(W)\right)=H_{c k} .
\end{aligned}
$$

Finally, because the $J_{c}$ are chosen "smoothly" as $c$ varies over $B$, one can show the $H_{c}$ give a "smooth" choice.

It is easy to see that this connection on $\mathfrak{B}_{k}$ can be extended uniquely to a connection on $\mathfrak{B}$ by the use of right translation. Since $J_{c}\left(H_{c}\right) \subset H_{c}$ if $c \in \mathfrak{B}_{k}$ and since (i) holds, condition $(\alpha)$ is satisfied also on $\mathfrak{B}$. $(\beta)$ is satisfied because of the way the connection was constructed.

In order to prove the uniqueness of the connection, suppose $H^{\prime}$ is a connection on $\mathfrak{B}$ satisfying $(\alpha)$ and $(\beta)$. If $c \in B_{k},(\beta)$ implies that $H_{c}^{\prime} \subset W$, and therefore $J\left(H_{c}^{\prime}\right) \subset J(W)=W$. But $(\alpha)$ implies that $J\left(H_{c}^{\prime}\right)=H_{c}^{\prime}$ so that $H_{c}^{\prime} \subset W \cap W^{\prime}=H_{c}$. Since $\operatorname{dim}\left(H_{c}^{\prime}\right)=m=\operatorname{dim}\left(H_{c}\right), H_{c}^{\prime}=H_{c}$. Thus the connection $H^{\prime}$ restricted to $\mathfrak{B}_{k}$ equals $H$ restricted to $B_{k}$. Since the extension to $\mathfrak{B}$ is unique, $H^{\prime}=H$.

3. An example. Let $M$ be a complex analytic manifold of complex dimension $r$ and let $\mathfrak{B}$ be the bundle of complex bases over $M$, so that $B=\left(p ; e_{1}, \cdots, e_{n}\right)$ where $p \in M$ and $e_{1}, \cdots, e_{n}$ is a complex basis of $M_{p}$. In this case, $\mathfrak{B}$ is a principal bundle with structural group $G$ the complex linear group. $G$ is the complexification of the unitary group $K$. $B$ is a complex analytic manifold. A complex coordinate neighborhood $U(\varepsilon)$ of the point $\left(p ; e_{1}, \cdots, e_{r}\right)$ is obtained by first choosing complex coordinates $z_{1}, \cdots, z_{r}$ about $p$; then

$$
e_{i}=\sum_{j=1}^{r} a_{i j} \frac{\partial}{\partial z_{j}}, \operatorname{det}\left(a_{i j}\right) \neq 0,
$$

and $\left(q, f_{1}, \cdots, f_{r}\right) \in U(\epsilon)$ if $\left|z_{i}(q)\right|<\varepsilon$ and $\left|b_{i j}-a_{i j}\right|<\varepsilon$ where

$$
f_{i}=\sum_{j=1}^{r} b_{i j} \frac{\partial}{\partial z_{j}} .
$$

The coordinates of points in $U(\varepsilon)$ are $\widetilde{z}_{1}, \cdots \widetilde{z}_{r}$, $\tilde{z}_{i j}$ where $z_{i}\left(q, f_{1}, \cdots, f_{i}\right)=$ $z_{i}(q)$ and $z_{i j}\left(q, f_{1}, \cdots, f_{r}\right)=b_{i j}$. It is easy to check that the operation of $G$ on $B$ preserves the complex structure of $B$ so that (i) is true. Moreover the $z_{i j}$ 's restricted to a fiber give the complex structure of $G$, i.e., (ii) also holds. The reduction of $G$ to the unitary group $K$ is equivalent to making $M$ into a Hermitian space [7]; in this case $B_{k}=\left(p, e_{i}, \cdots, e_{r}\right)$ where $e_{i}, \cdots, e_{r}$ are complex orthonormal relative to the hermitian inner product on $M_{p}$.

The example just described is the one that arises naturally in the 
study of complex manifolds. The reduction of the bundle of complex bases to the unitary group gives rise to a connection usually described in terms of a coordinate system. For example, see [6]. That this connection is the same as the one in $\S 2$ can be seen by checking that $(\alpha)$ and $(\beta)$ hold and using the uniqueness result.

If $M$ is a hermitian manifold, the real part of the hermitian inner product on $M_{p}$ makes $M$ into a Riemannian manifold. We have the following bundles over $M: \mathfrak{B}^{1}$, the principal bundle of all real bases with $G^{1}$ the real linear group in $2 r$ dimensions as structural group; $B^{2}$, the principal subbundle of orthogonal bases with $G^{2}$ the real orthogonal group as structural group; $B^{3}$, the principal bundle of complex bases with $G^{3}$ the complex linear group in $r$ dimensions as structural group; and $B^{4}$ the principal bundle of complex orthogonal bases with $G^{4}$, the unitary group as structural group. The mapping

$$
\left(f_{1}, \cdots, f_{r}\right) \rightarrow\left(f_{1}, \cdots, f_{r}, i f_{1} \cdots, i f_{r}\right)
$$

maps the set of complex bases of a vector space into the set of real bases. Consequently, we can think of $B^{3}$ as a subbundle of $B^{1}$ and $B^{3} \cap B^{2}=B^{4}$.

Let $\omega_{j}$ be the complex valued 1 -form on $B^{3}$ which assigns to $t \in B_{\left(m, f_{1}, \ldots, f_{r}\right)}^{3}$ the $j$ th component of $\pi(t)$ relative to the complex basis $f_{1} \cdots f_{r}$ at $M_{m}$. Then $\omega_{j}\left(J_{b}(t)\right)=i \omega_{j}(t)$ so that $\omega_{j}$ is of type $(1,0)$. In fact, since $B^{3}$ is complex analytic, locally $\omega_{j}=\Sigma=U_{j k} d z_{k}$ with $U_{j k}$ holomorphic functions so that $d \omega_{j}$ is of type $(2,0)$. Let $\omega_{j k}$ be the complex valued 2 -forms which our connection gives rise to. Condition $(\alpha)$ insures that $\omega_{j k}$ is also of type $(1,0)$ so that the torsion form $d \omega_{j}-\Sigma \omega_{k} \wedge \omega_{k j}$ is of type $(2,0)$. Condition $(\beta)$ tells us that on $\mathfrak{B}^{4} \omega_{k j}=$ $-\bar{\omega}_{j k}$, for our connection restricted to $\mathfrak{B}^{4}$ is a connection on $\mathfrak{B}^{4}$. Consequently [3], the vanishing of the torsion of our connection is equivalent to the Kähler condition on the Hermitian metric. The extension of our connection to $\mathfrak{B}^{2}$ will then also have zero torsion and must therefore be the Riemannian connection. We have proved the following.

CoRollary. If $M$ is a Kähler manifold, then the Riemannian connection can be restricted to the bundle of complex bases and there coincides with the connection constructed in $\$ 2$.

4. The curvature. We return to the general case. Let $\omega$ denote the one form with values in $g$ associated with the connection $H$ on $\mathfrak{B}$ and let $\Lambda$ be the curvature form.

THEOREM. If $B$ is a complex manifold, then for any tangent vectors $s$ and $t$ at $b \in B, \Lambda(s, t)=\Lambda\left(J_{b}(s), J_{b}(t)\right)$. 
Proof. Let $\mathfrak{S}_{b}$ denote the projection of $B_{b}$ on the horizontal space $H_{b}$. Then we know [1] that $A(s, t)=d \omega\left(\mathfrak{S}_{b} s, \mathfrak{S}_{l} t\right)$. Let $\tilde{s}$ and $\tilde{t}$ denote horizontal vector fields on $B$ for which $\tilde{s}(b)=\mathfrak{S}_{b}(s)$ and $\tilde{t}(b)=\mathfrak{S}_{b}(t)$. Since $\tilde{s}$ and $\tilde{t}$ are horizontal, so are $J(\tilde{s})$ and $J(\tilde{t})$. Since $B$ is a complex manifold $[J \tilde{s}, J \tilde{t}]-[\tilde{s}, \tilde{t}]=J\{[J \tilde{s}, \tilde{t}]+[\tilde{s}, J \tilde{t}]\}$. See [4] for example. Finally, since $J_{b}$ leaves vertical and horizontal spaces of $B_{b}$ invariant, $J_{b} \mathfrak{S}_{b}=\mathfrak{S}_{b} J_{b}$. We can now compute:

$$
\begin{aligned}
& \Lambda\left(J_{b}(s), J_{b}(t)\right)-\Lambda(s, t)=d \omega\left(\mathfrak{S}_{b} J_{b}(s), \mathfrak{S}_{b} J_{b}(t)\right)-d \omega\left(\mathfrak{S}_{b}(s), \mathfrak{S}_{b}(t)\right) \\
& \quad=d \omega\left(J_{b} \mathfrak{S}_{b}(s), J_{b} \mathfrak{F}_{b}(t)\right)-d \omega\left(\mathfrak{S}_{b}(s), \mathfrak{S}_{b}(t)\right) \\
& \quad=d \omega(J \tilde{s}(b), J \tilde{t}(b))-d \omega(\tilde{s}(b), \tilde{t}(b)) \\
& \quad=\frac{1}{2}\left\{-\omega\left([J \tilde{s}, J \tilde{t}]_{b}\right)+\left.J \tilde{s}(\omega(J \tilde{t}))\right|_{b}-\left.J \tilde{t}(\omega(J \tilde{s}))\right|_{b}\right. \\
& \left.\quad+\omega\left([\check{s}, \tilde{t}]_{b}\right)-\left.\tilde{s}(\omega(\tilde{t}))\right|_{b}+\left.\tilde{t}(\omega(\tilde{s}))\right|_{b}\right\} .
\end{aligned}
$$

Since $\omega$ annihilates horizontal vectors, this last expression equals

$$
-\frac{1}{2} \omega\left([J \tilde{s}, J \tilde{t}]_{b}-[\tilde{s}, \tilde{t}]_{b}\right)=-\frac{1}{2} \omega\left(J\left\{[J \check{s}, \tilde{t}]_{b}+[\tilde{s}, J \tilde{t}]_{b}\right\}\right) .
$$

However, for any tangent vector $u$,

$$
\omega(J u)=\omega\left(\mathfrak{S}_{b} J u+\left(I-\mathfrak{S}_{b}\right) J u\right)=\omega\left(\left(I-\mathfrak{S}_{b}\right) J u\right)=\omega\left(J\left(I-\mathfrak{S}_{b}\right) u\right) .
$$

But condition $(\alpha)$ implies that this last term equals $i \omega\left(\left(I-\mathfrak{S}_{b}\right) u\right)=i \omega(u)$. We conclude, therefore, that

$$
\Lambda\left(J_{b}(s), J_{b}(t)\right)-\Lambda(s, t)=-\frac{1}{2} i\left\{\omega\left([J \tilde{s}, \tilde{t}]_{b}+[s, J \tilde{t}]_{b}\right)\right\} .
$$

Suppose that $b \in B_{k}$. Since $\tilde{s}, \tilde{t}, J \tilde{s}$, and $J \tilde{t}$ are horizontal, they are tangent to $B_{k}$ at points of $B_{k}$. Therefore, $[J s, \tilde{t}]_{b}$ and $[\tilde{s}, \tilde{J t}]_{b}$ are also tangent to $B_{k}$. As a consequence of condition $(\beta)$ the range of $\omega$ restricted to $\left(B_{k}\right)_{b}$ is $\mathfrak{f}$. Hence the right side of equation (A) lies in $i$. On the other hand, since $b \in B_{k}, \Lambda(s, t)=\Lambda\left(\mathfrak{S}_{0}(s), \mathfrak{S}_{b}(t)\right) \in \mathfrak{f} ;$ similarly, $\Lambda\left(J_{b}(s), J_{b}(t)\right) \in \mathfrak{f}$. Therefore the left side of equation (A) lies in $\mathfrak{f}$. But $\mathfrak{i} \cap i^{\sharp}=0$ so that both sides of $(\mathrm{A})$ are zero.

Thus we have proved the desired formula for $b \in B_{k}$. Since $\Lambda$ is equi-invariant under right translation by $g \in G$ and since any $c \in B$ is of the form $b g$ for some $g \in G$, the formula holds throughout B.

Since $\omega\left(J_{b} u\right)=i \omega(u), w$ is a connection of type $(1,0)$. Also

$$
\begin{aligned}
\Lambda\left(J_{b} s, t\right)+\Lambda\left(s, J_{b} t\right) & =\Lambda\left(J_{b} s, t\right)+\Lambda\left(J_{b} s, J_{b}^{2} t\right) \\
& =\Lambda\left(J_{b} s, t\right)-\Lambda\left(J_{b} s, t\right)=0
\end{aligned}
$$

so that the curvature form is of type $(1,1)$. 


\section{REFERENCES}

1. W. Ambrose and I. M. Singer, A theorem on holonomy, Trans, Amer. Math Soc., 75 (1953), 428-443.

2. M. F. Atiyah, Complex analytic connections in fibre bundles, Trans. Amer. Math. Soc., 85 (1957), 181-207.

3. Shiing-shen Chern, Characteristic classes of hormitian manifolds, Ann. of Math., 47 (1956), 85-121.

4. Alfred Frölicher, Zur Differentialgeometrie der komplexen Sirurturen, Math. Ann., 129 (1955), 50-95.

5. K. Kodaira and D. C. Spencer, On deformations of complex analytic siructures I, Ann. of Math., 67 (1958), 328-402.

6. S. Nakano, On complex analyitic vector bundles, J. Math. Soc. Japan, 7 (1955), 1-12.

7. Norman Steenrod, The topology of fibre bundles, Princeton (1951). 


\section{PACIFIC JOURNAL OF MATHEMATICS}

\section{EDITORS}

\section{David Gilbarg}

Stanford University

Stanford, California

\section{R. A. Beaumont}

University of Washington

Seattle 5 , Washington

\author{
A. L. Whiteman
}

University of Southern California Los Angeles 7, California

L. J. Paige

University of California

Los Angeles 24, California

\author{
E. F. BECKENBACH \\ C. E. BURGESS \\ E. HEWITT \\ A. HORN
}

\author{
V. GANAPATHY IYER \\ R. D. JAMES \\ M. S. KNEBELMAN \\ L. NACHBIN
}

ASSOCIATE EDITORS
I. NIVEN

T. G. OSTROM

H. L. ROYDEN

M. M. SCHIFFER
E. G. STRAUS

G. SZEKERES

F. WOLF

K. YOSIDA

\section{SUPPORTING INSTITUTIONS}

\author{
UNIVERSITY OF BRITISH COLUMBIA \\ CALIFORNIA INSTITUTE OF TECHNOLOGY \\ UNIVERSITY OF CALIFORNIA \\ MONTANA STATE UNIVERSITY \\ UNIVERSITY OF NEVADA \\ OREGON STATE COLLEGE \\ UNIVERSITY OF OREGON \\ OSAKA UNIVERSITY \\ UNIVERSITY OF SOUTHERN CALIFORNIA
}

\author{
STANFORD UNIVERSITY \\ UNIVERSITY OF TOKYO \\ UNIVERSITY OF UTAH \\ WASHINGTON STATE COLLEGE \\ UNIVERSITY OF WASHINGTON \\ * * * \\ AMERICAN MATHEMATICAL SOCIETY \\ CALIFORNIA RESEARCH CORPORATION \\ HUGHES AIRCRAFT COMPANY \\ SPACE TECHNOLOGY LABORATORIES
}

Mathematical papers intended for publication in the Pacific Journal of Mathematics should be typewritten (double spaced), and the author should keep a complete copy. Manuscripts may be sent to any one of the four editors. All other communications to the editors should be addressed to the managing editor, L. J. Paige at the University of California, Los Angeles 24, California.

50 reprints per author of each article are furnished free of charge; additional copies may be obtained at cost in multiples of 50 .

The Pacific Journal of Mathematics is published quarterly, in March, June, September, and December. The price per volume (4 numbers) is $\$ 12.00$; single issues, $\$ 3.50$. Back numbers are available. Special price to individual faculty members of supporting institutions and to individual members of the American Mathematical Society: $\$ 4.00$ per volume; single issues, $\$ 1.25$.

Subscriptions, orders for back numbers, and changes of address should be sent to Pacific Journal of Mathematics, 2120 Oxford Street, Berkeley 4, California.

Printed at Kokusai Bunken Insatsusha (International Academic Printing Co., Ltd.), No. 6, 2-chome, Fujimi-cho, Chiyoda-ku, Tokyo, Japan.

PUBLISHED BY PACIFIC JOURNAL OF MATHEMATICS, A NON-PROFIT CORPORATION

The Supporting Institutions listed above contribute to the cost of publication of this Journal, but they are not owners or publishers and have no responsibility for its content or policies. 


\section{Pacific Journal of Mathematics}

\section{Vol. 9, No. $2 \quad$ June, 1959}

Lee William Anderson, On the breadth and co-dimension of a topological lattice

Frank W. Anderson and Robert L. Blair, Characterizations of certain lattices

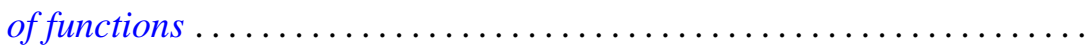

Donald Charles Benson, Extensions of a theorem of Loewner on integral

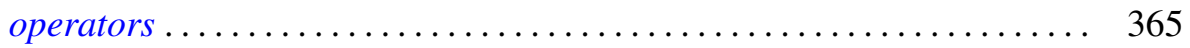

Errett Albert Bishop, A duality theorem for an arbitrary operator ........ 379

Robert McCallum Blumenthal and Ronald Kay Getoor, The asymptotic distribution of the eigenvalues for a class of Markov operators ........

Delmar L. Boyer and Elbert A. Walker, Almost locally pure Abelian

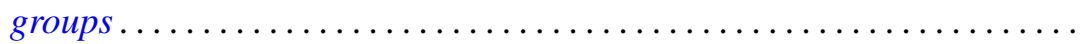

Paul Civin and Bertram Yood, Involutions on Banach algebras ........... Lincoln Kearney Durst, Exceptional real Lehmer sequences .... 415

Eldon Dyer and Allen Lowell Shields, Connectivity of topological

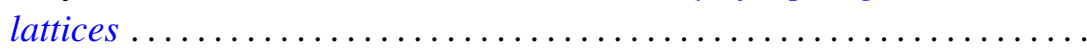

Ronald Kay Getoor, Markov operators and their associated

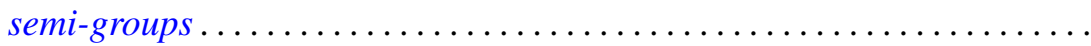

Bernard Greenspan, A bound for the orders of the components of a system of algebraic difference equations

Branko Grünbaum, On some covering and intersection properties in

Minkowski spaces ............................

Bruno Harris, Derivations of Jordan algebras ..............

Henry Berge Helson, Conjugate series in several variables.

Isidore Isaac Hirschman, Jr., A maximal problem in harmonic analysis.

II .

Alfred Horn and Robert Steinberg, Eigenvalues of the unitary part of a matrix

Edith Hirsch Luchins, On strictly semi-simple Banach algebras ...

William D. Munro, Some iterative methods for determining zeros of

functions of a complex variable...

John Rainwater, Spaces whose finest uniformity is metric .

William T. Reid, Variational aspects of generalized convex functions ....

A. Sade, Isomorphisme d'hypergroupoï des isotopes ...... . .

Isadore Manual Singer, The geometric interpretation of a special

connection . . .

Charles Andrew Swanson, Asymptotic perturbation series for characteristic

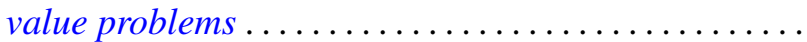

